\title{
Fitting an Epidemiological Model to Transmission Dynamics of COVID-19
}

\author{
Endalew Getnet Tsega \\ Department of Mathematics, College of Science, Bahir Dar University Bahir Dar, Ethiopia
}

\author{
Article Info \\ Keywords: Covid-19, Epidemic, Model \\ parameters, Reported data, SIRD \\ model, Worldwide. \\ 2010 AMS: 97N40, 93A30, 92D30. \\ Received: 26 July 2020 \\ Accepted: 24 December 2020 \\ Available online: 29 December 2020
}

\begin{abstract}
A rapid increase in daily new cases was reported in the world from February 19 to April 3, 2020. In this study, a susceptible-infected-recovered-dead (SIRD) was developed to analyse the dynamics of the global spread of COVID-19 during the above-mentioned period of time. The values of the model parameters fitted the reported data were estimated by minimizing the sum of squared errors using the Levenberg-Marquardt optimization algorithm. A timedependent infection rate was considered. The set of differential equations in the model was solved using the fourth order Runge-Kutta method. It was observed that a time-dependent parameter gives a better fit to a dynamic data. Based on the fitted model, the average value of basic reproduction number $(R O)$ for COVID-19 trasmission was estimated to be 2.8 which shows that the spread of COVID-19 disease in the world was growing exponentially. This may indicate that the control measures implemented worldwide could not decrease the COVID-19 transmission.
\end{abstract}

\section{Introduction}

First cases of COVID-19 were reported at the end of 2019 in Wuhan city of China [1]. It has been rapidly spreading in the world and affecting the lives of millions of people, the global economy and educational systems. Governments have been taking drastic measures to stop the transmission of the disease. The control measures include tracing close contacts, promoting social distancing, imposing lockdown, quarantining the infected cases and self-protection using protective equipment. Unfortunately, these measures have not been able to stop the spreading of COVID-19, which caused several outbreaks around the globe [2, 3, 4]. Many studies have been done to understand the dynamics of the COVID-19 disease in different regions or countries to create prevention awareness among people and support healthcare authorities in taking appropriate control measures $[3,4,5,6]$. Compartmental models are commonly used to describe the spread of infectious disease including COVID-19 [3, 7, 8]. In these models, the population under study is subdivided into a number of compartments based on infection status. The flows from one compartment to another are described by ordinary differential equations (or difference equations) [9, 10, 11]. The parameters in the differential equations are determined by fitting the model to available data using optimization methods [12, 13].

The novel coronavirus (COVID-19) daily new cases was increasing dramatically in the world from February 19 to April 3, 2020. The objective of this study was to develop a compartmental (SIRD) model describing this phenomenon mathematically. An exponential function was intended to characterize the number of infected cases [14]. The data source to the COVID-19 cases for this study was Worldometer [15].

\section{Mathematical model}

Figure 2.1 shows the COVID-19 daily new cases from February 1 to May 10, 2020. The increment was very high from February 19 to April 3, 2020. The cases were 516 on February 19 and 96352 on April 3. The increment was relatively continuous during this time. In this study, a deterministic SIRD model $[4,9]$ was used to describe the dynamics of COVID-19 spread.

The model divides the world population into four separate compartments: Susceptible (S), Infected (I), Recovered (R) and Dead (D). The underlying assumptions (constant population size and well-mixed population) of SIR model of Kermack and MacKendrick [16] were 


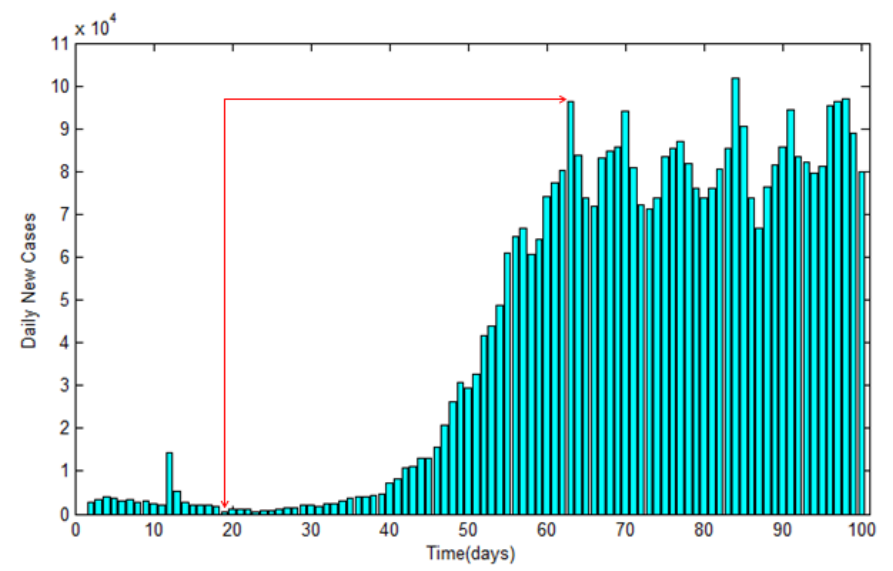

Figure 2.1: COVID-19 daily new cases in the world from February 1 to May 10, 2020.

considered. The control measures being taken were not considered in the modeling. The system of differential equations in SIRD model is given by

$$
\begin{gathered}
\frac{d S}{d t}=-\frac{\alpha}{N} S I \\
\frac{d I}{d t}=\frac{\alpha}{N} S I-\beta I-\gamma I \\
\frac{d R}{d t}=\beta I \\
\frac{d D}{d t}=\gamma I
\end{gathered}
$$

where the parameters $\alpha, \beta$ and $\gamma$ represent the infection rate, recovery rate and death rate respectively. The total confirmed cases $T$ is calculated as $T=I+R+D$. In this model, the parameter $\alpha$ was consider to be time-dependent [3, 4] and expressed by an exponential function as

$$
\alpha(t)=A\left(1-e^{-k t^{n}}\right)
$$

where $A, k$ and $n$ are parameters to be determined. The basic reproduction number, $R_{0}$ (the number of persons that an infected number will infect ) is estimated from the SIRD model as [17]:

$$
R_{0}=\frac{\alpha}{\beta+\gamma}
$$

In epidemiology, the basic reproduction number is considered to be very important to describe the spreading nature of a disease [12].

\section{Estimation of model parameters}

The model parameters were estimated by minimizing the sum of squared errors [18, 19]

$$
\operatorname{SSE}(A, k, n, \beta, \gamma)=\|Y-\hat{Y}\|
$$

where

is the matrix of the COVID-19 data for compartments,

$$
Y=\left[\begin{array}{cccc}
S\left(t_{1}\right) & I\left(t_{1}\right) & R\left(t_{1}\right) & D\left(t_{1}\right) \\
S\left(t_{2}\right) & I\left(t_{2}\right) & R\left(t_{2}\right) & D\left(t_{2}\right) \\
\vdots & \vdots & \vdots & \vdots \\
S\left(t_{n}\right) & I\left(t_{n}\right) & R\left(t_{n}\right) & D\left(t_{n}\right)
\end{array}\right]
$$

$$
\hat{Y}=\left[\begin{array}{cccc}
S\left(\hat{t}_{1}\right) & I\left(\hat{t}_{1}\right) & R\left(\hat{t}_{1}\right) & D \hat{\left(\hat{t}_{1}\right)} \\
S\left(\hat{t}_{2}\right) & I\left(\hat{t}_{2}\right) & R \hat{\left(\hat{t}_{2}\right)} & D \hat{\left(t_{2}\right)} \\
\vdots & \vdots & \vdots & \vdots \\
S\left(\hat{t}_{n}\right) & I\left(\hat{t}_{n}\right) & \text { hat } R\left(t_{n}\right) & D \hat{\left(t_{n}\right)}
\end{array}\right]
$$

is the matrix of the corresponding estimates predicted by the model and \|\| is the Euclidean norm. The MATLAB function ode45 was used to solve the model differential equations in Eq.(2.1)-Eq.(2.4). The MATLAB function lsqnonlin with Levenberg-Marquardt algorithm was used for minimization of SSE. Based on Worldometer, the world population was taken to be $N=778359130$. The initial estimates of the parameters $A, \beta$ and $\gamma$ were calculated by replacing the derivatives in Eq.(2.1), Eq.(2.3) and Eq.(2.4) by forward difference approximation and employing least squares parameter estimation using respective data and time step size of $\Delta t=1$ day [9]. A MATLAB code was developed to estimate the values of all parameters with the given range and to display the results of this study. 


\section{Results and discussion}

Figure 4.1 displays the SIRD model results of COVID-19 in the world from February 19 to April 3, 2020. It compares the actual data with the infected, recovered, dead and total cases predicted by the model as $[4,19]$. From the best fit optimization, the values of the model parameters $\alpha, \beta$ and $\gamma$ were estimated to be $0.09572,0.02793$ and 0.006268 , respectively. The values of the parameters $A, k$ and $n$ used in the exponential model of the infection rate were also obtained as $0.1414,0.00008087$ and 3.4446, respectively. Using Eq.(2.6) and the model parameters $\alpha, \beta$ and $\gamma$, the average value of basic reproduction number $(R O)$ was estimated to be 2.8. This indicates that COVID-19 was spreading exponentially in the world population [14]. The model results showed that all the infected, recovered and dead cases were increasing during the period of time. As we can see in Figure 4.1, COVID-19 daily new cases for upcoming 37 days (from April 4 to May 10, 2020), has different distribution from the previous days. Thus, the model cannot be used to forecast the distribution for the upcoming days.

The predicted graphs of the infected cases and total confirmed cases are almost similar. We can observe that the resulting SIRD model prediction agreed well with the actual data for all four cases. The relative error of the model fit $[18,20]$ to the data for total confirmed cases is shown in Figure 4.2. The time-dependent infection rate gave a better fit to the reported COVID-19 data.

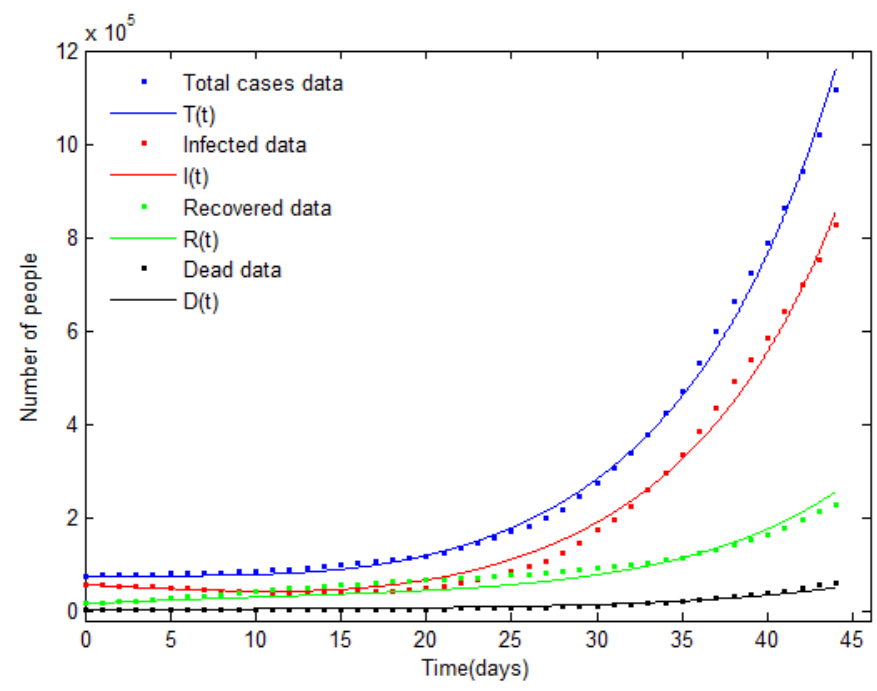

Figure 4.1: The SIRD model fitted to COVID-19 reported data from February 19 to April 3, 2020.

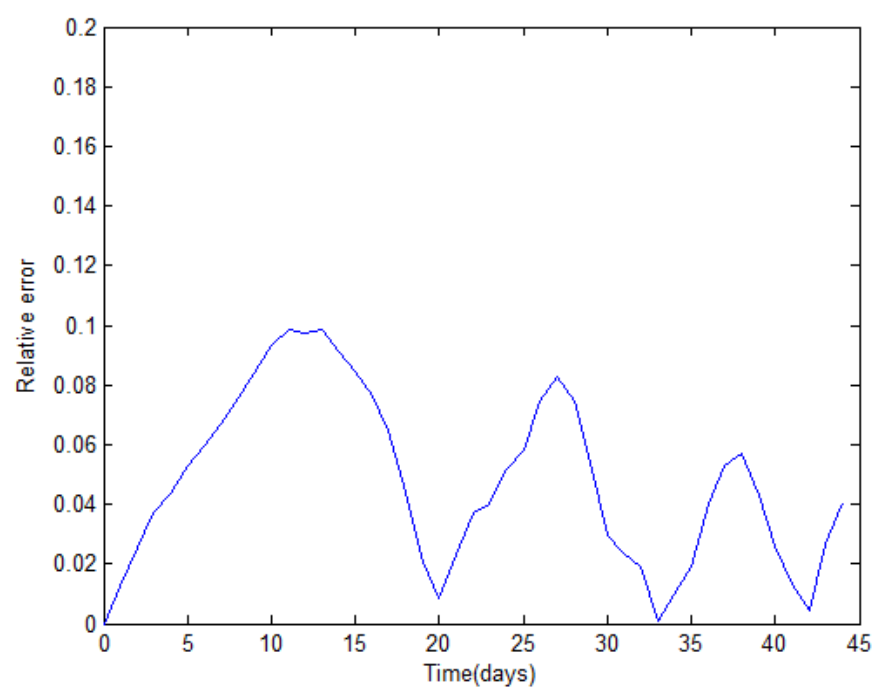

Figure 4.2: Plot of the relative error between the reported data and results of SIRD model for total confirmed cases.

\section{Conclusion}

In this study, SIRD epidemiological model was fitted to worldwide COVID-19 reported data from February 19 to April 3, 2020 . A time-dependent infection rate was considered. Model parameters were estimated using nonlinear least squares fit by minimizing the sum of squared errors. The model results ensured that COVID-19 was spreading exponentially in the world population during this time. The number of infected cases and total confirmed cases showed similar distributions. This study may indicate that the transmission of COVID-19 was not slow down by the control measures implemented globally. The application of mathematics in describing real phenomena may be appreciated. 


\section{Conflict of interest statement}

The author declares that there is no conflict of interest regarding the publication of this article.

\section{References}

[1] World Health Organization,Coronavirus disease (COVID-19) outbreak, 2020, available at https://www.who.int/health-topics/Coronavirus.

[2] A. J. Kucharski, T. W. Russell, C. Diamond, Y. Liu, J. Edmunds, S. Funk, R. M. Eggo, F. Sun, M. Jit, J. D. Munday, N. Davies, Early dynamics of transmission and control of COVID-19: a Mathematical modeling study, The Iancet infectious diseases, 2020.

[3] A. L. Kapetanovic, D. Poljack, Modeling the epidemic outbreak and dynamics of COVID-19 in Croatia, (2020), pp.arXiv-2005.

[4] D. Caccavo,Chinese and Italian COVID-19 outbreaks can be correctly described by a modified SIRD model, available at https://doi.org/10.1101/2020.03.19.20039388.

[5] F. Ndairou, I. Area, J. J. Nieto, D.F. Torres,Mathematical modeling of COVID-19 transmission dynamics with a case study of Wuhan, Chaos, Solitons Fractals, (2020), p.109846.

[6] R. Sameni, Mathematical modeling of epidemic diseases; a case study of the COVID-19 coronavirus, (2020), arXiv:2003.11371.

[7] J. C. Blackwood, L. M. Childs, An introduction compartmental modeling for the budding infectious disease modeller, Letters in Biomathematics, 5(1)(2018), 195-221.

[8] E. L. Piccolomiini, F. Zama, Monitoring Italian COVID-19 spread by an adaptive SEIRD model, medRxiv, 2020.

[9] C. Anastassopoulou, L. Russo, A. Tsakris, C. Siettos, Data-based analysis, modelling and forecasting of the COVID-19 outbreak, PloS one, 15(3) (2020), p.e0230405.

[10] P. Khrapov, A. Loginova, Mathematical modeling of the dynamics of coronavirus COVID-19 epidemic development in China, Int. J. Open Inf. Technol., 8(4)(2020), 13-16.

[11] F. Brauer, C. Castillo-Chavez, Z. Feng, Mathematical Model in, Epidemiology, Springer, New York, 2019

[12] M. Y. Li, An Introduction to Mathematical Modeling Infectious Diseases, (Vol.2) Cham: Springer, 2018.

[13] M. A. A. Al-qaness, A. A. Ewees, H. Fan, M. E. Abd Elaziz, Optimization methods for Forecasting confirmed cases of COVID-19 in China, J. Clinical Medicine, 9(3) (2020), pp.674.

[14] J. Fernandez-Villaverde, C. I. Jones, Estimating and Simulating a SIRD Model of COVID-19 for Many Countries, States and Cities(No.w27128), National Bureau of Economic Research, 2020.

[15] https://www.worldometers.info/coronavirus/.

[16] W. O. Kermack, A. G. MacKendrick, A contribution to the mathematical theory of epidemics, In: Proceedings of the Royal Society of London, vol. A, p. 700-721, 1927

[17] L. Ferrari, G. Gerardi, G. Manzi, A. Micheletti, F. Nicolussi, S. Salini, Modeling provisional COVID-19 epidemic data in Italy using an adjusted time-dependent SIRD model, (2020), arXiv :2005.12170.

[18] E. L. Piccolomiini, F. Zama, Monitoring Italian COVID-19 spread by an adaptive SEIRD model, medRxiv, 2020.

[19] A. Ianni, Rossi, N., Describing the COVID-19 Outbreak Fitting Modified SIR models to Data, medRxiv, 2020.

[20] G. Chowell, Fitting dynamic models to epidemic outbreaks with quantified uncertainty: A primer for parameter uncertainty, identifiability, and forecasts. Infectious Disease Modelling, 2(2017), 379-398. 